\title{
Über Kathepsin und Phosphoproteid-Phosphatase in der löslichen Fraktion aus Hirngewebe von Maus und Ratte')
}

\author{
Von E. Albert ${ }^{2}$ ) \\ Aus dem Laboratoire de Morphologie Animale, Université libre de Bruxelles (Direktor: Professor Jean Bracbet)
}

(Eingegangen am 25. Mai 1967)

\begin{abstract}
In der löslichen Fraktion aus Mäuse- und Rattenhirn lassen sich nebeneinander die Aktivitäten von Kathepsin, Phosphoproteid-Phosphatase und Saurer Phosphatase nachweisen. Substrat von Kathepsin und Phosphoproteid-Phosphatase sind Phosphoproteide, welche im Gehirn lebhaft umgesetzt werden. Sie spalten auch Peptid- und Esterbindungen von Casein. Beide Fermente unterscheiden sich in ihrem Verteilungsmuster je nach Reifegrad des Organs und Hirriteils. Während des Aufbaus und der Differenzierung der nervösen Struktur in den ersten Lebenswochen der Maus steigt zuerst die Phosphoproteid-Phosphatase an, welche in nervenzellreichen Gebieten auch später überwiegt. Kathepsinaktivitäten treten dagegen bedeutend später und ganz allmählich auf und überwiegen in an Leitungsbahnen reichen Hirnteilen. Die Saure Phosphatase folgt nur in der zeitlichen Entwicklung der Phosphoproteid-Phosphatase, nicht aber in der lokalen Verteilung, so daß es sich bei dieser phosphatabspaltenden Aktivität um ein eigenes Ferment handeln dürfte.

Cathepsin, phosphoprotein phosphatase und acid phosphatase activities were demonstrated in the soluble fraction from mouse and rat brain. Phosphoproteins, which are vigorously metabolised in the brain, are substrates of cathepsin and a phosphoprotein phosphatase. They also attack the peptide and ester bonds of casein. Their relative quantities vary according to the maturation of the organ, and they are distributed differently within the brain. In the first few weeks after birth of the mouse, during the formation and differentiation of the nervous structure, there is first an increase in phosphoprotein phosphatase which also later predominates in those regions rich in nerve cells. Cathepsin increases much later and gradually, and becomes predominant in those parts of the brain where nerve fibres are concentrated. The acid phosphatase follows the same time course of development as phosphoprotein phosphatase, but does not have the same local distribution, so they must be different enzymes.
\end{abstract}

Die Untersuchung von Hirngewebe ist schwierig wegen seiner dichten Permeabilitätsschranke, der Empfindlichkeit gegen Sauerstoffmangel und des komplexen Aufbaus aus verschiedenartigen Zellelementen zusammen mit der hohen funktionellen Differenzierung kleinster Areale. Dennoch ließen die mit unterschiedlichen Untersuchungsmethoden gewonnenen Ergebnisse erkennen, daß Eiweißkörper hier sehr lebhaft umgesetzt werden und vornehmlich Ernährung und Funktion des Neurons gewährleisten $(1,2,3)$. Aus der Aktivität eiweißabbauender Fermente läßt sich ein Einblick in den Eiweißumsatz eines Gewebes $(4,5)$ gewinnen.

Phosphomonoesterasen sind zwar meist unspezifische Fermente, doch weist die Saure Phosphatase ${ }^{3}$ ) gewisse Beziehungen zum Eiweißstoffwechsel und zu den Proteasen auf. So kann z. B. Chymotrypsin neben Peptidbindungen auch Esterbindungen spalten (6). Auch kommt die saure Phosphatase vermehrt in den Orten aktiver Proteinsynthese vor, wie sezernierenden oder regenerierenden Zellen. Das Ferment aus Niere und aus Prostata ist spezifisch auf Phosphoproteide eingestellt (7).

Frühere Untersuchungen zur Verteilung der Sauren Phosphatase im Rinderhirn (8) hatten ergeben, daß dieses Ferment in der Hirnrinde so aktiv ist wie in der Leber und $\mathrm{da} \beta$ es sich in den durch Ultrazentrifugation gewonnenen Hirngewebsfraktionen in der löslichen Fraktion anreichert. Während sich das Cytoplasmapräparat bei pH 5,6 gegenüber 13 biologisch wichtigen Phosphatverbindungen als inaktiv erwies, griff es als einziges

1) Herrn Professor KLENK zum 70. Geburtstag in Verehrung gewidmet.

2) Ausgeführt mit Mitteln der Deutschen Forschungsgemeinschaft. 3) Der Trivialname Saure Phosphatase wird hier gebraucht für das Enzym Orthophosphoric monoester phosphohydrolase EC.3.1.3.2, Chymotrypsin für Peptide peptidohydrolase EC 3.4.4.5, Phosphoproteid-Phosphatase für Phosphoric monoester hydrolase EC 3.1.3 und Kathepsin für Peptide peptidohydrolase EC 3.4.4.
Substrat Casein an. Das Gehirn enthält zwar kein Casein, wohl aber native Phosphorproteide vom gleichen Polyphosphoryl-Seryltyp wie Casein oder Phosvitin (9), angereichert in den membranreichen Fraktionen $(10,11)$. Die Rolle dieser relativ hoch phosphorylierten Eiweißkötper, die abwechselnd phosphoryliert und dephosphoryliert werden, wird in einer Beteiligung am Kationentransport während der nervösen Erregungsleitung vermutet $(10,11)$. Man kennt heute im Cytoplasma des Hirngewebes zwei Fermente, die Phosphoproteide aufspalten, aber an verschiedenen Stellen des Moleküls angreifen.

Das eine ist eine Phosphoproteid-Phosphatase, die sich zu $60 \%$ in der löslichen Fraktion befindet und aus Phosphoproteiden wie Casein oder Phosvitin Phosphat abspaltet. Dagegen erwies sie sich 14 anderen physiologischen Phosphatverbindungen gegenüber als inaktiv. Das Ferment aus Ochsenhirn ist besonders aktiv in den Hirnteilen mit hoher respiratorischer Aktivität, vor allem der Hirnrinde, und zeichnet sich durch ein $\mathrm{pH}$ Optimum von 5,5 und $\mathrm{K}_{\mathrm{m}}=3,74 \mathrm{mg}$-Atome CaseinPhosphor $(12,13)$ aus.

Der intrazelluläre Eiweißabbau wird im Tierkörper von Gewebs-Proteasen besorgt, welche im schwach sauren Milieu wirken und die Peptidbindungen spalten. Ihrer verdauenden Wirkung wegen werden sie Kathepsine genannt (14). Man vermutet, daß sie physiologische Bedeutung vorwiegend für die Transpeptidation (6) haben. Im Kalbshirn zeigte sich Kathepsin, auf Eiweiß bezogen, $12 \mathrm{mal}$ so aktiv wie im Muskel (15). Besonders reichlich fand es sich im Grau der Großhirnhemisphären und des Kleinhirns $(16,17)$. Kathepsin greift ebenfalls vorzugsweise Casein, aber auch andere Phosphoproteide an. Daneben vermochte es nur noch aus Insulin und Ribonuclease Trichloressigsäure-lösliches, Tyrosin-positives Material freizusetzen. Im Rattenhirn fand sich das Kathepsin ebenfalls in der löslichen Fraktion von $0,25 \mathrm{M}$ 
Tab. 1

Optimale Bedingungen für die Aktivitätsbestimmung von Kathepsin und Phosphoproteid-Phosphatase

\begin{tabular}{|c|c|c|}
\hline Substrat & $\begin{array}{l}\text { Kathepsin } \\
3 \% \text { Casein nach HAMMARSTEN } \\
\text { in } 8 M \text { Harnstofflösung }\end{array}$ & $\begin{array}{c}\text { Phosphoproteid-Phosphatase } \\
1 \% \text { Casein isoelectric }\end{array}$ \\
\hline \multirow{2}{*}{$\begin{array}{l}\text { Inkubationsvolumen (ml) } \\
\text { Optimale Fermentkonzentration (mg Frischgewicht/ml lösliche Fraktion } \\
\text { aus Hirngewebe) } \\
\text { pH-Optimum } \\
\text { Optimale Inkubationszeit (Min.) } \\
\text { Optimale Substratkonzentration } \\
\text { Michaeliskonstante }\end{array}$} & 0,32 & 1,2 \\
\hline & $\begin{array}{c}58,6 \\
5,4 \\
60 \\
6,0 \mathrm{mg} \text { Casein } / \mathrm{ml} \\
1,47 \mathrm{mg} / \mathrm{ml}\end{array}$ & $\begin{array}{c}10,4 \\
5,6 \\
30 \\
2,2 \mu \mu \mathrm{Mol} \text { Casein-P/ml } \\
0,33 \cdot 10^{-3} \mathrm{M} \text { Gesamt-Casein-P }\end{array}$ \\
\hline
\end{tabular}

Saccharosehomogenat und war durch ein pH-Optimum von $7,1-7,3$, optimale Substratkonzentration von $1-2 \mathrm{mg}$ Casein pro $3,2 \mathrm{ml}$ Inkubationsansatz, ein $\mathrm{V}^{1}$ ) von $10 \mathrm{nMol}$ Tyrosinäquivalenten pro $\mathrm{Min}$. und $\mathrm{Ca}^{++}$ Abhängigkeit (18) ausgezeichnet.

In der vorliegenden Arbeit wurde der Plan verfolgt, die Aktivitäten der drei Fermente Saure Phosphatase, Phosphoproteid-Phosphatase und Kathepsin in der löslichen Fraktion aus Hirngewebe nebeneinander zu erfassen und ihre Änderung in Abhängigkeit von der Hirnreifung und Hirnlokalisation zu beobachten.

\section{Methodik}

Bereitung der löslichen Fraktion aus Mäuse- und Rattenhirn

Weiße Mäuse aus der Zucht des Euratom-Centre in Moll/Belgien wurden durch Nackenelongation getötet, das Hirn entnommen und im Kühlraum auf Eis weiterverarbeitet. Nach Bestimmung des Feuchtgewichtes wurde das gesamte Mäusehirn mit 7 Teilen 10 proz. eiskalter Saccharoselösung im Glashomogenisator nach Potter-Elvehjem homogenisiert. Alle Glasgeräte sowie die Saccharoselösung wurden zuvor sterilisiert. Das Homogenat wurde $60 \mathrm{Min}$. in der Spinco-Ultrazentrifuge Modell L mit Rotor 40,2 bei 40000 U./Min. (101000 g) zentrifugiert. Der klare, leicht gelblich gefärbte Überstand wurde als lösliche Fraktion und Enzympräparat benützt. Die für die Untersuchung der Abhängigkeit der Enzymaktivitäten in verschiedenen Hirnregionen vom Alter der Tiere bzw. vom Reifegrad des Hirns benutzten Mäuse waren 6 Stdn. bis 420 Tage alt. Je 4-5 Tiere bildeten eine Altersgruppe. Von jedem Mäusehirn wurde einzeln die lösliche Fraktion hergestellt. Weiße Ratten bezogen wir vom Händler. 13 Tiere hielten wir bereits ein halbes Jahr, 7 über ein Jahr im hiesigen Tierstall. Die Gewichte der jüngeren Tiere lagen um $250 \mathrm{~g}$, die der älteren um $400 \mathrm{~g}$. Die Tiere wurden in vier Gruppen zu je fünf Tieren aufgeteilt, wobei eine Gruppe nur aus über einjährigen Tieren bestand. Die Ratten wurden dekapitiert und von den Hirnen auf Eis vier Teile abgetrennt: das gesamte Kleinhirn, der Hypothalamus, flache Rindenschnitte der Großhirnrinde und der Hirnstamm caudal vom oberen Brückenrand. Die Hirnteile von je 5 Tieren wurden vereinigt und aus diesem pool jeweils die lösliche Fraktion hergestellt.

Bestimmung der Fermentaktivität

Saure Phosphatase

Die Aktivität wurde mit Phenolphthalein-Diphosphat (Natriumsalz) als Substrat bestimmt. Die Farbintensität von freigesetztem Phenolphthalein wurde in alkalischem Milieu bei $540 \mathrm{~nm}$ gemessen. Die Methode von Huggins und Talalay (19) wurde für ein Inkubations-Volumen von $2 \mathrm{~m} l$ modifiziert.

\section{Kathepsin}

Kathepsin bestimmten wir mit einer modifizierten Methode nach Duspiva (20). Der Ansatz von $0,32 \mathrm{~m} i$ enthielt $0,15 \mathrm{~m} l$ lösliche Fraktion (Enzym) und 2,1 mg Casein nach HAMMarsten.

1) Abkürzungen: $\mathrm{K}_{\mathrm{m}}=$ Michaeliskonstante; $\mathrm{V}=$ Reaktionsgeschwindigkeit bei Substratsättigung; $\mathrm{PhE}=$ Kolorimetrisches Aquivalent für $1 \mu \mathrm{g}$ Phenolphthalein, freigesetzt während Inkubation von 60 Min. bei $38^{\circ}$ und $\mathrm{pH} 5,6$. Ablesungsvolumen $4 \mathrm{ml}$.
Das säurelösliche, Tyrosin-positive Material wurde im Überstand der Trichloressigsäure-Fällung mit der Folin-Reaktion gemessen (21). In Leerwerten wurde die Menge Tyrosin-positiven Materials bestimmt, das während der Inkubation aus dem Ferment bzw. Substrat freigesetzt wurde. Proben von Tyrosin-Standards wurden parallel bestimmt.

\section{Phosphoproteid-Phosphatase}

Substrat: Eine 5 proz. Lösung von Casein isoelectric (Difco Laboratories, Detroit/USA, P-Gehalt $0,82 \%$ ) wird durch Jösen unter Alkalizusatz ( $\mathrm{pH}$ 6,9) hergestellt. Hieraus wird eine 1 proz. Caseinlösung von $\mathrm{pH}$ 5,6 bereitet, indem mit Acetat-Veronalpuffer $\mathrm{pH}$ 5,2 nach Micbaelis (22) aufgefüllt wird.

Der Ansatz von $1,2 \mathrm{~m} l$ enthielt $0,1 \mathrm{~m} l$ lösliche Fraktion (Enzym) und $10 \mathrm{mg}$ Cascin isoelectric.

Im säurelöslichen Uberstand nach Trichloressigsäurefällung wurde das anorganische Phosphat nach KutrNeR und LichtensteIn (23) bestimmt. Die aus Enzym bzw. Substrat während der Inkubation freigesetzte Phosphatmenge wurde in Leerwerten ermittelt. Ebenso wurden Proben von P-Standards parallel bestimmt.

\section{Ergebnisse und Diskussion}

Die Untersuchung von Fermentaktivitäten in der löslichen Fraktion aus Mäuse- und Rattenhirn ergab, $\mathrm{da}$ sich hier regelmäßig Kathepsin findet. Dieses spaltet Casein in niedermolekulare Peptide. Das gleiche partikelfreie Cytoplasmapräparat enthält eine PhosphoproteidPhosphatase, welche Phosphat aus Casein freisetzt.

Als Vergleichsgröße zur Aktivität von Kathepsin und Phosphoproteid-Phosphatase wurde wegen ihrer erwähnten Beziehungen zum Eiweißstoffwechsel auch die Saure Phosphatase in der löslichen Fraktion gemessen. Sie war stets sehr aktiv. In den insgesamt untersuchten 128 Mäusehirnen fanden wir je nach Reifegrad 16,67 bis 26,67 $\mathrm{PhE} / \mathrm{Min}$. pro $\mathrm{m} l$ löslicher Fraktion. Alle drei Fermente fanden sich entsprechend den Befunden von Guroff (18), Heald (11) und Rose $(12,13)$ in der löslichen Fraktion. Obwohl mitunter auch katheptische Aktivität in den Mitochondrien $(24,25)$ und Lysosomen (26) beschrieben wurde, ist doch bei der hier geübten schonenden Homogenisierung im Glashomogenisator und in 10proz. Saccharoselösung der Austritt von Fermenten aus Partikeln oder Lysosomen nicht wahrscheinlich.

Zuerst wurden für die beiden Phosphoproteid-spaltenden Fermente Kathepsin und Phosphoproteid-Phosphatase die optimalen Bedingungen für die Bestimmung ihrer Aktivität ermittelt. Die Studien wurden an der unveränderten löslichen Fraktion angestellt, also an ungereinigten Fermenten, was die Aussagekraft der gewonnenen kinetischen Daten einschränkt; dennoch können die Werte einen gewissen Einblick in die Eigenart der beiden Fermentaktivitäten (Tab. 1) gewähren. 
Unter diesen optimalen Bedingungen geprüft können beide Fermente, ausgedrückt als relative Aktivitäten, in verschiedener Hinsicht miteinander verglichen werden.

Um Einblick zu gewinnen, ob die Aktivitäten der getesteten Fermente eng aneinander gekoppelt auftreten oder ob sie selbständig und unabhängig voneinander wirken, wurde ihre Veränderung mit wachsendem Reifegrad des Mäusehirns sowie ihre Verteilung in unterschiedlichen Ratten-Hirnregionen verfolgt. Es zeigte sich, daß die Altivitäten der drei Fermente in verschiedenen Altersstufen unterschiedlich verteilt sind (Abb. 1).

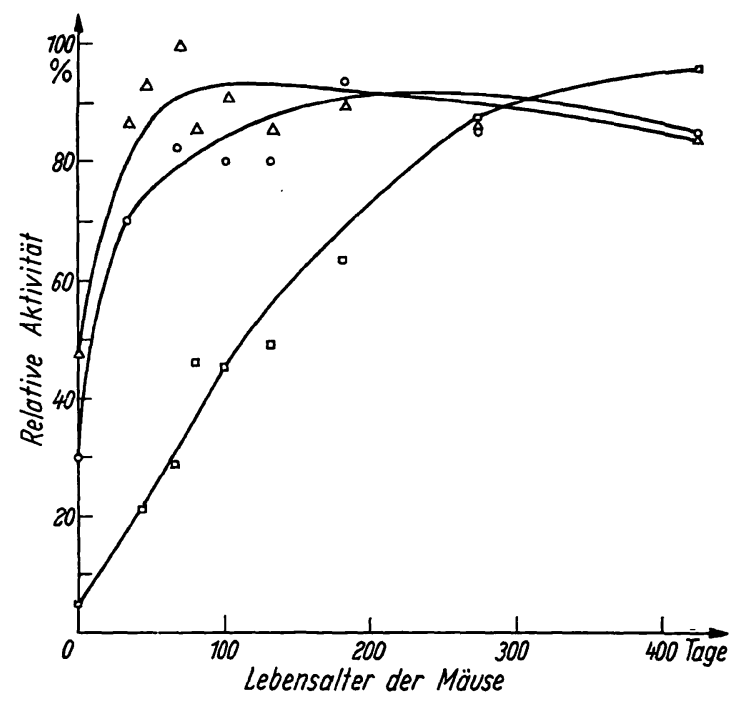

Abb. 1

Aktivitäten von Saurer Phosphatase, Phosphoproteid-Phosphatase und Kathepsin in der löslichen Fraktion aus Mäusehirnhomogenat in Abhängigkeit vom Reifegrad des Hirns.

Jede Altersgruppe besteht aus 4-5 Tieren, deren Hirne einzeln geprüft wurden. Relative Aktivität $=\%$ der Maximalaktivität pro $\mathrm{m} l$ lösliche Fraktion:

Saure Phosphatase $(0): 100 \%=1600 \mathrm{PhE} / 60 \mathrm{Min}$

Phosphoproteid-Phosphatase $(\Delta): 100 \%=9,67 \cdot 10^{-3} \mu \mathrm{Mol} \mathrm{P} / \mathrm{Min}$ Kathepsin ( $\square): 100 \%=11,50 \cdot 10^{-3} \mu$ Mol Tyrosinäquiv./Min.

Die Phosphoproteid-Phosphatase ist schon in den ersten Stunden nach der Geburt deutlich nachweisbar. Ihre Aktivität steigt rasch weiter an und erreicht ihre volle Höhe nach 2-3 Monaten.

Die Saure Phosphatase läuft mit ihr konform.

Die Aktivität des Kathepsins nimmt nur sehr langsam zu. Sie erreicht höhere Werte erst nach 6 Monaten, nimmt aber auch dann noch stetig weiter zu. Das liegt durchaus noch im aufsteigenden Schenkel der Lebenskurve bei einer Lebenserwartung der Maus von bis zu drei Jahren. Bei vier Wochen alten Mäusen ist das Ferment unter den hier eingehaltenen Bedingungen wohl meßbar, beträgt aber nur ein Sechstel der später erreichten Aktivität, während zu diesem Zeitpunkt die Phosphoproteid-Phosphatase bereits $80 \%$ ihrer beobachteten maximalen Aktivität zeigt.

Die Untersuchung dieser Fermentaktivitäten in funktionell verscbiedenen Hirnteilen ist am Rattenhirn leichter durchführbar, da es größer ist. Die beiden Fermente zeigen hier die gleichen kinetischen Eigenschaften wie

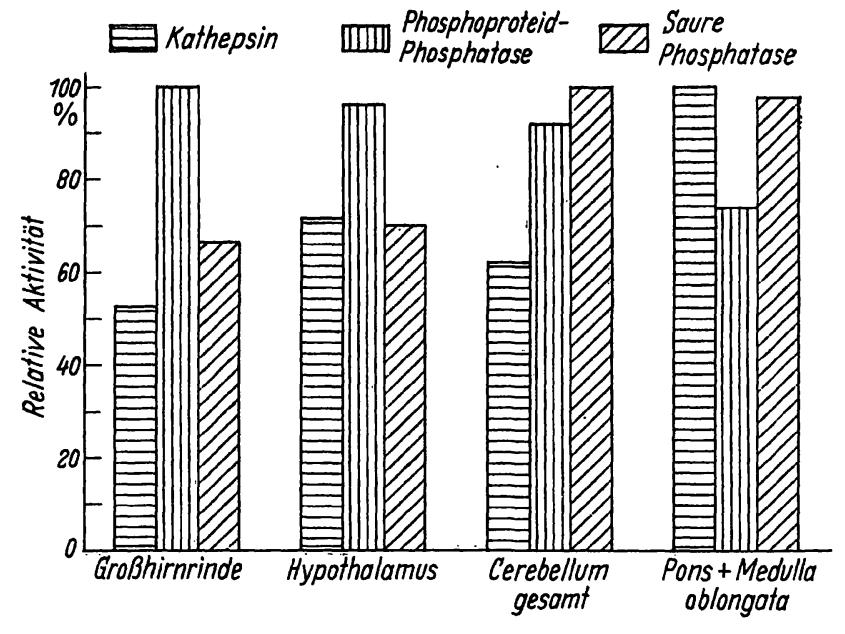

Abb. 2

Aktivitäten von Saurer Phosphatase, Phosphoproteid-Phosphatase und Kathepsin in der löslichen Fraktion aus 4 verschiedenen Rattenhirnregionen

Relative Aktivität $=\%$ der Maximalaktivität pro $\mathrm{ml}$ lösliche Frak-

Saure Phosphatase: $100 \%=2000 \mathrm{PhE} / 60 \mathrm{Min}$.

Phosphoproteid-Phosphatase: $100 \%=8,00 \cdot 10^{-3} \mu \mathrm{M}$ ol P/Min. Kathepsin: $100 \%=12,83 \cdot 10^{-3} \mu \mathrm{Mol}$ Tyrosinäquiv./Min. Die mittlere Abweichung vom Mittelwert lag bei $10-12 \%$

im Mäusehirn. Es ergab sich in den vereinigten Hirnteilen von je 5 Hirnen in allen vier Tiergruppen übereinstimmend, daß Kathepsin und PhosphoproteidPhosphatase innerhalb gewisser Grenzen unterschiedlich lokalisiert sind (Abb. 2). So ist in der Großhirnrinde die Aktivität an Phosphoproteid-Phosphatase hoch und liegt in der Medulla oblongata deutlich niedriger, was mit den Ergebnissen von Rose (12) übereinstimmt. Das Kathepsin dagegen findet sich nur in relativ geringer Aktivität in der Hirnrinde, ist dagegen im Pons-MedullaPräparat fast doppelt so hoch angereichert. Die Saure Phosphatase läuft hier konform mit dem Kathepsin. Hypothalamus und Kleinhirn, die stärker gemischt aus weißen Faserzügen und grauen Zentren aufgebaut sind, zeigen ein weniger unterschiedliches Fermentmuster.

Ein Vergleich der Aktivitäten der drei geprüften Fermente unter verschiedenen Bedingungen ergibt, daß sie nicht parallel laufen. Sie scheinen vielmehr zu einem funktionellen Muster angeordnet zu sein.

Die Hirnrinde ist zellreich und enthält die Masse der großen Nervenzellen mit ihren Dendriten und dem Beginn des Neuriten. Beim neugeborenen und jungen Tier, bei dem sich die Zellen differenzieren und ihre Fortsätze ausreifen, ist die Phosphoproteid-Phosphatase bereits hoch aktiv. Sie überwiegt auch später weiterhin in den nervenzellreichen Gebieten, die zur Versorgung des Neuriten anhaltend Eiweiß̧ aufbauen.

Das Pons-Medulla-Gebiet dagegen enthält zwar auch graue Kerne, die aber an Menge zurücktreten hinter den Leitungsbahnen, deren flüssiger Inhalt sich gerade im Überstand nach Ultrazentrifugation ansammeln dürfte. Hier überwiegt das Kathepsin. Die Aktivität dieses Ferments nimmt nur langsam zu und erreicht lebhaftere Aktivität erst bei 6 Monate alten Mäusen. Möglicherweise ist erst dann die funktionelle Reife mit Verwertung 
der Informationen in Höchstgeschwindigkeit vollendet. Die Saure Phosphatase ist beim ganz jungen Tier bereits deutlich aktiv wie die Phosphoproteid-Phosphatase, zeigt sich aber stärker konzentriert in Hirnteilen, die reich an Leitungsbahnen sind, worin sie dem Kathepsin folgt. Die hier erfaßte phosphatabspaltende Aktivität - geprüft an einer körperfremden Phosphatverbindung - ist nicbt die mit anderer Methodik nachgewiesene Aktivität der Phosphoproteid-Phosphatase, sondern zeigt ein eigenes, charakteristisches Verhalten von Lokalisation und Aktivitätsverlauf.

Aus dem unterschiedlichen Verhalten der im Hirncytoplasma enthaltenen Aktivitäten von Kathepsin, Phosphoproteid-Phosphatase und Saurer Phosphatase schließen wir, daß es sich um drei wohlunterschiedene, voneinander unabhängige Fermente handelt. Untersuchungen $z u$ ihrer Isolierung und Charakterisierung sind im Gange.

\title{
Literatur
}

1. Ruchter, D., Brit. Med. J., 1255 (1959). - 2. WAELSCH, H., in: Metabolism of the nervous system, Hrsg. D. Richter Pergamon Press, London (1957). - 3. MÉrer, F. T. und F. Gallyas, J. Neurochem. 11, 257 (1964). - 4. POPE, A., J. Neurochem. 4, 32 (1959). - 5. Siebert, G., A. Schmitr und R. von Malortie, Hoppe-Seyler's Z. physiol. Chem. 342, 20 (1965). - 6. Haurowirz, F., Ann. New York Acad. Sci. 68, 3 (1957). - 7. Sundararajan, T. A. und P. S. SARMA, Biochem. J. 56, 125 (1954). - 8. AlBerT, E., Hoppe-Scyler's Z. physiol. Chem. 302, 129 (1955). - 9. Rodnighr, R., in: Protides of the Biological Fluids, Hrsg. H. Peeters, S. 39. Elsevier Publ., Amsterdam (1966). - 10. Rabinowitz, M. und F. LipManN, J. biol. Chemistry 235, 1043 (1960). 11. Heald, P. J., Phosphorus Metabolism in Brain. Pergamon Press, Oxford (1960). - 12. Rose, S. P. R., Biochem. J. 83, 614 (1962). - 13. Rose, S. P. R. und P. J. Heald, Biochem. J. 81, 339 (1961). - 14. WILLSTÄtTER, R. und E. BaMANN, Hoppe-Seyler's Z. physiolog. Chem. 180, 127 (1929). - 15. KIES, M. W. und S.
Schwimmer, J. biol. Chemistry 145, 685 (1942). - 16. Ansell, G. B. und D. Richrer, Biochim. biophysica Acta (Amsterdam) 13, 87 (1954). - 17. Palladin, A. V., N. M. Polyakova und V. K. Lrshko, J. Neurochem. 10, 187 (1963). - 18. Guropr, G., J. biol. Chemistry 239, 149 (1964). - 19. Huggins, C. und P. TALALAY, J. biol. Chemistry 159, 399 (1945). - 20. Duspiva, F., Protoplasma 32, 211 (1939). - 21. Lowry, O. H., N. J. Rosebrough, A. L. FarR und R. J. Randall, J. biol. Chemistry 193, 265 (1951). 22. Mrchaelis, L., Biochem. Z. 234, 139 (1931); Docum. Geigy, Wiss. Tab., 6. Aufl., S. 277 Basel (1960). - 23. Kutrner, Th. und L. Lichtenstein, J. biol. Chemistry 86, 671 (1930). - 24. La JTHA, A., in: Regional Biochemistry, Hrsg. S. S. Kety und J. Elkes, Pergamon Press, Oxford (1961). - 25. Polyakova, N. M., Y. V. BeLrK und L. A. SARYuK, Ukr. Biokhim. Zh. 32, 623 (1960). zit nach 17. - 26. Benufax, H., A. M. Berleur und A. Doyen, Biochem. J. 66, 32 (1957).

\section{Enzymatische Hydrolyse von Diäthyl-p-nitrophenylphosphat (E 600) durch menschliches Serum}

\author{
Von K. KrrsCH \\ Aus dem Pbysiologisch-Cbemischen Institut der Justus Liebig-Universität Gießen (Direktor: Prof. Dr. Hj. Staudinger)
}

(Eingegangen am 14. Juni 1967)

Es wird ein einfacher, kontinuierlicher photometrischer Test zur Messung der enzymatischen Hydrolyse von E 600 durch menschliches Serum angegeben.

Kinetische Grundlagen, Michaelis-Konstanten, pH-Optimum und Normalwertè werden beschrieben.

A simple, continuous, photometric test is described for the measurement of the enzymic hydrolysis of $\mathrm{E} 600$ by human serum. The kinetic basis of the test, Michaelis constants, the $\mathrm{pH}$ optimum and normal values are described.

Beim Zusatz von Serum zu einer wäßr. E 600-Lösung tritt eine rasch zunehmende Gelbfärbung auf. Diese ist auf Freisetzung von p-Nitrophenol durch enzymatische Hydrolyse des E 600 nach folgender Gleichung zurückzuführen:

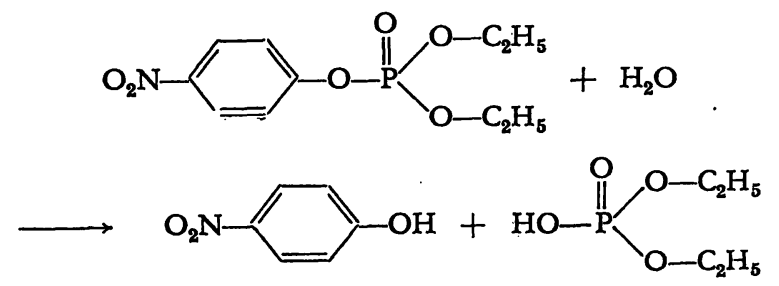

Als Meßgröße dient p-Nitrophenol, das. bei $405 \mathrm{~nm}$ im kontinuierlichen photometrischen Test direkt in der
Küvette gemessen werden kann (s. Methodik). Nach einer Einteilung von AldRIDGE $(1,2)$ bezeichnet man Carboxylesterasen, welche durch organische Phosphorsäureester nicht gehemmt werden, als A-Esterasen ${ }^{1}$ ). Nach AldRIDGe ist die E 600-spaltende Aktivität des Serums mit der aromatische Ester (wie z. B. p-Nitrophenylacetat) hydrolysierenden sog. A-Esterase identisch. Sie läßt sich jedoch von der Procain-, Atropin- und Pseudocholinesterase abgrenzen (2). Das Vorkommen von A-Esterasen im Serum verschiedener Tierarten ist

1) Der Trivialname A-Esterase wird hier gebraucht für das Enzym Arylester-Hydrolase, EC 3.1.1.2.; B-Esterase für Carboxylesterase EC 3.1.1.1; Procain-, Atropin- und Pseudocholinesterase für Cholinesterase EC 3.1.1.8. 